\title{
Analysis of UAV image Quality using Edge Analysis
}

\author{
Pyung-Chae Lim ${ }^{1}$, Taejung Kim ${ }^{1 *}$, Sang-Il Na², Kyung-Do Lee², Ho-Yong Ahn² , Jaeyoung Hong ${ }^{3}$ \\ ${ }^{1}$ Dept. of Geoinformatic Engineering, Inha University, 100 Inharo, Namgu, Incheon, Korea - vudco88@inha.edu, tezid@inha.ac.kr \\ ${ }^{2}$ National Institute of Agricultural Sciences, Rural Development Administration - (Sangi1917, kdlee11, hyahn85) @korea.kr \\ ${ }^{3}$ KEVA DRONE CO., Ltd., 99 Daehak-ro, Yuseong-gu, Daejeon, Korea - hiy@ kevadrone.com
}

Commission IV, WG IV/3

KEY WORDS: UAV, GRD, Image Quality Analysis, NIIRS, Edge detection, MTF,

\begin{abstract}
:
UAVs (Unmanned aerial Vehicles) can acquire images easily without large cost. For this reason, use of UAV is spreading to diverse fields such as orthoimages and DEM/DSM production. The spatial resolution of images is usually expressed as a GSD (Ground Sampling Distance). The GSD from UAV has higher performance than other platforms such as satellites and aircraft because it shoot at low altitude. However, blurring and noise may occur on UAV images due to the weather and the stability of UAV. However, since the GSD from UAV cannot sufficiently meet the spatial resolving power of the actual image system, a criterion for determining the spatial resolution of image is needed. Therefore we emphasize that the quality of the image needs to be analysed. Actual performance indicators such as GRD (Ground Resolved Distance) and NIIRS (National Image Interpretability Rating Scales), which can be measured through image analysis, are representative examples of image quality interpretation. It is possible to extract NIIRS form image quality related parameters such as MTF (Modulation Transfer Function), RER (Relative Edge Response) and SNR (Signal to Noise Ratio). In this paper, we aim to apply the Edge analysis method to UAV and to analyse the result. The analysis result showed that while GSD and NIIRS were highly dependent to imaging altitude, GRD and image sharpness showed optimal altitude ranges. The exact optimal range varied between images taken at different weather conditions. While we need a further study, this may indicate that edge analysis may provide an optimal operational altitude range suitable for the sensors.
\end{abstract}

\section{INTRODUCTION}

Along with the development of optical systems, various types of images are actively being distributed through various platforms such as aerial, satellite and UAVs (Unmanned aerial Vehicles). Accordingly, techniques for extracting and utilizing various information using various are developing, and a need for a scale for judging the quality of the image is also emphasized. In particular, UAV can acquire high quality images with high spatial resolution. For this reason, UAV are being used in various fields such as construction, agriculture, forestry, and disaster. However, the blurring or noise of the image may appear on the UAV due to weather or stability at the time of shooting. Also, the gap between the model of the UAV and the digital camera used is affected. In case when the image of the UAV is unclear about the image quality, acquisition and mapping of accurate three-dimensional information have a great influence on the work.

The spatial resolution of an image is usually expressed as GSD (Ground Sampling Distance). However, since the GSD of the unmanned airplane image does not indicate the level of spatial resolution capability of the real image system, a criterion for determining the resolution of the image is needed. In photogrammetry, the quality inspection of optical images is generally performed by edge analysis of acquired images.

In this case, MTF (Modulation Transfer Function), SNR (Signal to Noise Ratio) and GRD (Ground Resolved Distance) are the main quality factors to be calculated through the edge analysis, so that it is possible to grasp the performance represented by the actual platform and to find a suitable post-processing task when there is a problem. These quality factors are usually measured from artificial targets specially designed for edge analysis. These artificial targets are classified into various kinds according to the type of quality factor to be measured and the measurement method. The target mainly used for the edge analysis is like a tent of a black and white pattern having a specific reflectance difference.

In previous studies, various parameters such as NIIRS (National Image Interpretability Rating Scales), RER (Relative Edge Response), SNR, MTF and GRD were extracted using edge analysis algorithm and image quality analysis was performed using the extracted factors (Kim et al., 2010; Kim et al., 2011). In this paper, we apply the edge analysis method applied to satellite image quality test to UAV image and analyze the result. Actual performance indicators such as GRD and NIIRS, which can be measured through image analysis, are representative examples of image quality interpretation.

Unlike individual quality factors such as MTF, SNR, or GSD, NIIRS can represent the overall quality of an image and is a widely used quality indicator of metadata that is widely available as high-resolution satellite metadata such as IKONOS or QuickBird.

GRD is a representative index that can represent the resolution of an image. It is defined as the minimum distance between two objects identifiable in the image. It can be directly related to the interpretation or resolution of the image.

\section{EXPERIMENT PROCEDURE}

Figure 1 shows the process from image acquisition to Edge analysis for image quality analysis. In this paper, image quality analysis was performed through the process of establishing a photographing plan, image acquisition using Drones and the analysis of calculated parameters. 


\begin{tabular}{l}
\hline $\begin{array}{c}\text { Establish a } \\
\text { photographing plan }\end{array}$ \\
\begin{tabular}{|c|c|}
\hline $\begin{array}{c}\text { Image acquisition } \\
\text { using Drones }\end{array}$ \\
\hline $\begin{array}{c}\text { Edge analysis using } \\
\text { the software }\end{array}$ \\
\hline $\begin{array}{c}\text { Calculated parameter } \\
\text { analysis }\end{array}$ \\
\hline
\end{tabular}
\end{tabular}

Figure 1. Procedure for image quality analysis

\subsection{Establish a photographing plan}

In the establishing a photographing plan stage, we set up a shooting plan for the acquisition of images, and selected shooting areas, image sensors, and artificial targets for image analysis. For image acquisition, we selected a wide playground to shoot only artificial targets and the image sensor used was Sony A5100.

\begin{tabular}{|l|l|l|}
\hline & Sensor & SONY A5100 \\
\hline & Type & EO \\
\hline & Focal Length & $20 \mathrm{~mm}$ \\
\hline & Resolution & $6000 \times 4000$ \\
\hline & Sensor Dim & $23.5 \times 15.6 \mathrm{~mm}$ \\
\hline
\end{tabular}

Table 1. Image sensor specifications

Image acquisition was performed three times in total. The first and the second were acquired in the same place during the acquisition of three images. We were targeted artificial targets for accurate edge analysis.

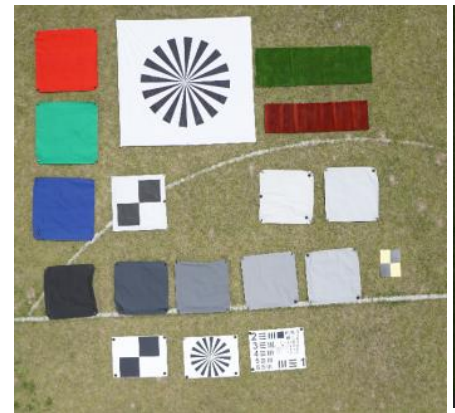

(a)

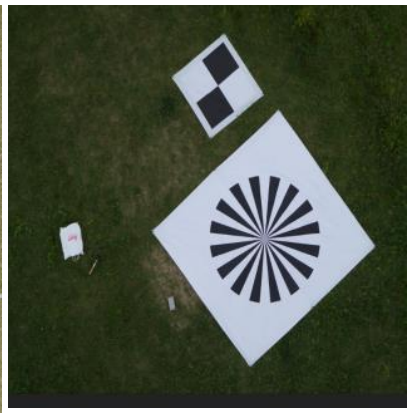

(b)
Figure 2. Experimental places for image acquisition : (a) first and second place; (b) third place

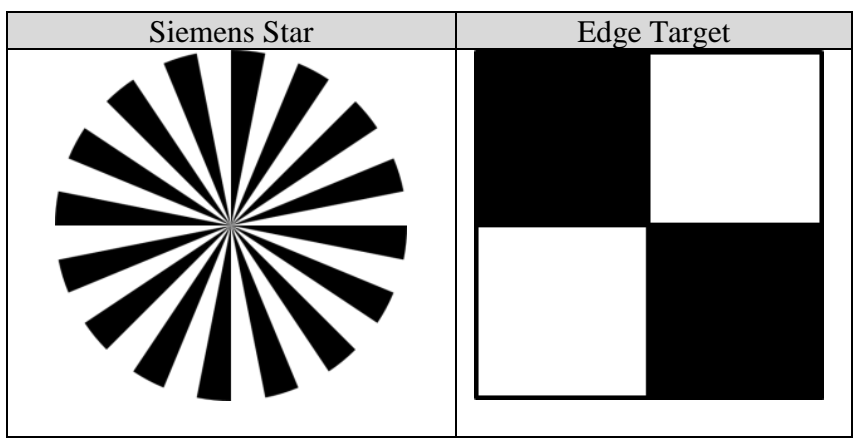

Table 2. Artificial target for edge analysis

\subsection{Image acquisition using Drones}

Rotor wing drones are capable of vertical takeoff and landing. It is also free to redirect and suitable for acquiring images at desired altitude and specific locations. In this experiment, we used a domestic rotor model to acquire the images at different altitudes. The images were acquired up to $150 \mathrm{~m}$ in intervals of $10 \mathrm{~m}$, and the GSD by altitude was calculated using Equation (1) for image quality analysis. The Pixel_size is one pixel size in the image, $\mathrm{H}$ is the altitude and $\mathrm{f}$ is the focal length of the sensor.

$$
G S D=\text { Pixel_size } \times H \div f
$$

\begin{tabular}{|l|l|l|}
\hline & Type & Coaxial Octocopter \\
\hline & Weight & $3.8 \mathrm{~kg}$ \\
\hline Flight Time & 20 minutes \\
\hline & Max speed & Greater than $15 \mathrm{~m} / \mathrm{s}$ \\
\hline Max Alt & Greater than $250 \mathrm{~m}$ \\
\hline & Play load & $2 \mathrm{~kg}$ \\
\hline Size & $960 \mathrm{~mm}$ \\
\hline & Battery & $6 \mathrm{~S}, 20,000 \mathrm{~m} \mathrm{Ah}$ \\
\hline
\end{tabular}

Table 3. Drone specification produced by KEVA DRONE

\subsection{Edge analysis}

In order to analyze the UAV image quality, the software originally developed for satellite images was applied to the UAV image. This software has shown that it can be applied not only to artificial artifacts but also to natural artifacts through previous researches (Kim, 2010; Kim, 2011). The software allows manual, semi-automatic extraction of edge information. Using this, NIIRS, GRD, SNR, RER and MTF were calculated. However, the edge points were extracted only for artifacts with clear brightness values for accurate image quality analysis.

\subsection{Calculated parameter analysis}

ESF (Edge Spread Function) can be estimated by edge analysis of images. The LSF (Line Spread Function) can be estimated by differentiating the ESF, and then the MTF can be calculated by Fourier transforming the LSF. Image quality parameters RER, SNR, MTF and GRD were calculated and analyzed by edge analysis..
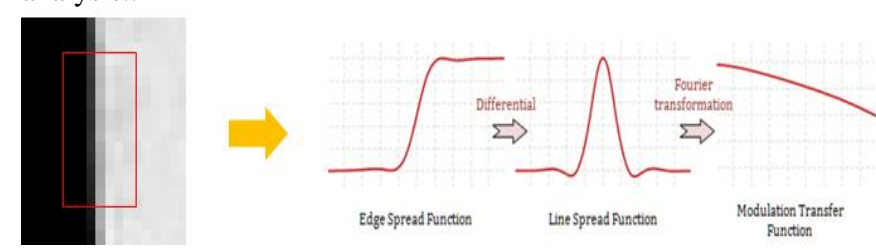

Figure 3. ESF, LSF and MTF

Using the parameters calculated by this software, NIIRS was calculated. NIIRS can be calculated by using GIQE (General Image Quality Equation) in Equation (2) (Leachtenauer et al., 1997). The higher the value, the sensor is excellent in performance.

$$
c_{0}+c_{1} \log _{10}(R E R)+c_{3} \frac{G}{S N R}+c_{4} H
$$

where RER = Relative Edge Response $\mathrm{G}=$ Noise Gain $\mathrm{H}=$ Overshoot Height $\mathrm{SNR}=$ Signal to Noise Ratio 


\begin{tabular}{|l|c|c|c|c|c|}
\hline & $c_{0}$ & $c_{1}$ & $c_{2}$ & $c_{3}$ & $c_{4}$ \\
\hline GIQE 3.0 & 11.810 & -3.320 & 3.320 & -1.000 & -1.480 \\
\hline $\begin{array}{l}\text { GIQE 4.0 (for } \\
\text { RER } \geq 0.9 \text { ) }\end{array}$ & 10.251 & -3.320 & 1.559 & -0.334 & -0.656 \\
\hline $\begin{array}{l}\text { GIQE 4.0 (for } \\
\text { RER < 0.9) }\end{array}$ & 10.251 & -3.160 & 2.817 & -0.334 & -0.656 \\
\hline
\end{tabular}

Table 4. Coefficient by GIQE version

The GRD of the image is defined as a relation between the shooting distance, the camera focal length, and the system resolution as shown in Equation (3) (Campbell et al, 2002).

$$
G R D=\frac{H}{f \times R}
$$

In this case, $\mathrm{R}$ is the system resolution, which means FWHM (Full Width at Half Maximum) indicating the width of the LSF curve. $\mathrm{H}$ is the imaging height of the image, and $\mathrm{F}$ is the focal length of the camera.

\section{EXPERIMENTAL RESULTS AND ANALYSIS}

\subsection{Visual inspection}

The image acquisition was performed three times in total. From the images acquired during the first shot, we could hardly extract the edge points from the image even at low altitudes due to very cloudy weather and erroneous camera settings. Figure 4 show the images from the first time at an altitude of $20 \mathrm{~m}$. It can be confirmed by visual inspection that most of the first drone shot images were blurred and the overall brightness was dark.

It was a sunny day at the second shooting and a cloudy day at the third shooting. As a result of the visual inspection, the images were clearer the first one. We could confirm that the third time drone shot images were a little darker because there was a lot of clouds at the time of shooting.
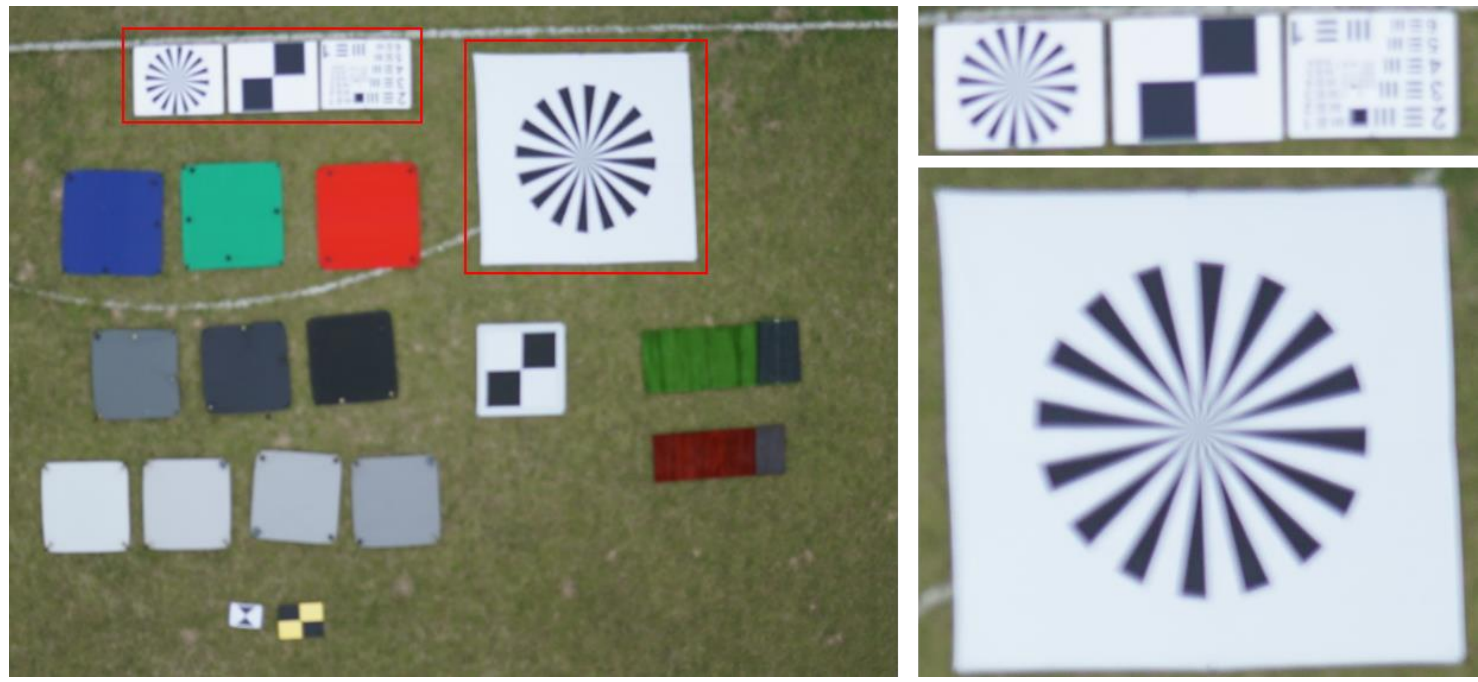

Figure 4. The artificial targets at $20 \mathrm{~m}$ altitude for edge detection in the first time drone shot

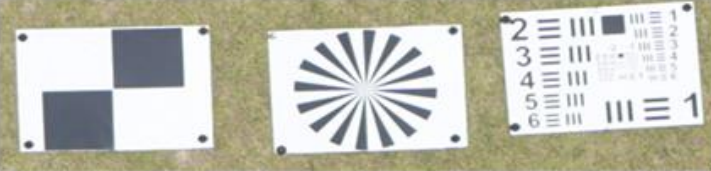

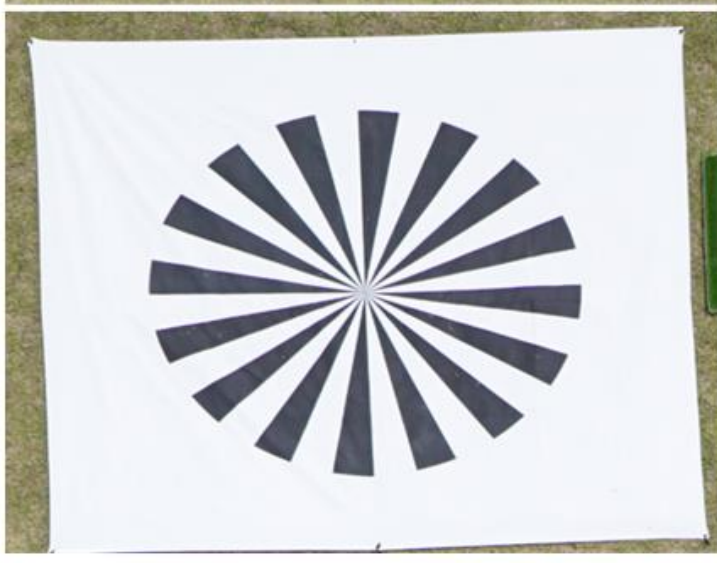

(a) Second time Drone shot at $20 \mathrm{~m}$

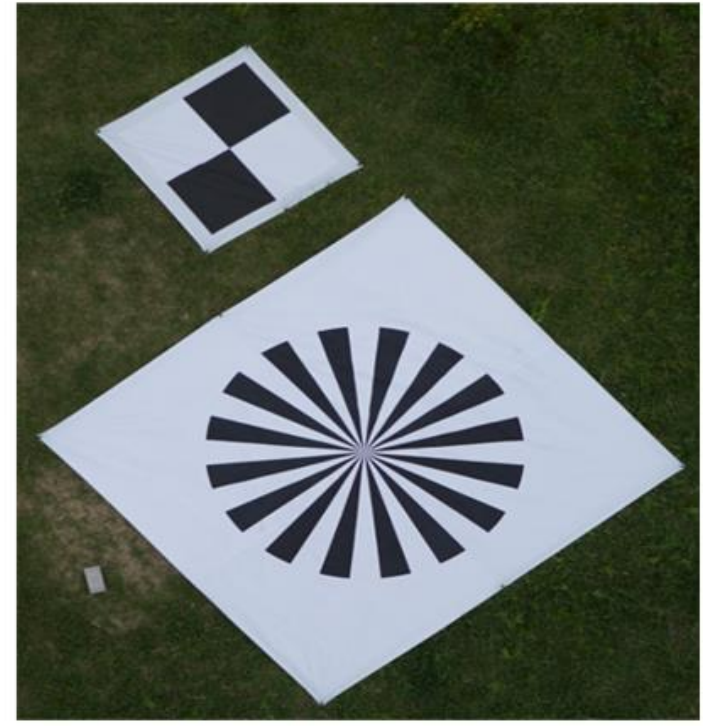

(b) Third time Drone shot at $20 \mathrm{~m}$

Figure 5. The second and third times drone shot at the same altitude 


\subsection{Edge detection for image analysis}

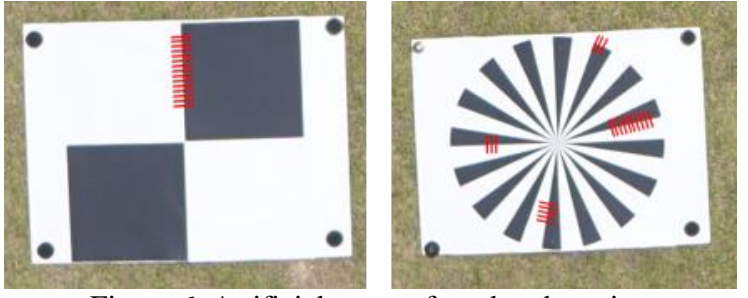

Figure 6. Artificial targets for edge detection

For image quality analysis, edge is detected at a point where the boundary of brightness is clear as shown in Figure 6. The ESF and LSF graphs are estimated through the detected edges as shown in Figure 8 and 9, and the image quality parameters are calculated. Figures 8 and 9 show graphs of the largest and smallest values of GRD in each table below (the yellow rows). The image quality was measured from individually estimated ESF through edge detection and averaged.

\subsection{Image quality analysis}

An artificial target was installed in the target area to acquire images of altitude at intervals of $10 \mathrm{M}$ and we calculated the parameters for image quality analysis. The difference between SNR, MTF and GRD values in Table 5 and 6 is large. This is considered that the weather during the flight test was very cloudy with large humidity and no sun light at third drone shot. These values are considered to be highly weather dependent. In the case of NIIRS, it can be seen in both tables that the value changes constantly according to altitude. This is due to the property that there are significant effects of the value of GSD in the NIIRS formula. The GSD is much better than the satellite image if the image is taken at low altitude using the drone. Because of this effect, NIIRS index was calculated to be as high as 9-11. In the both tables, it can be seen that GRD pixels are better at $30 \sim 100 \mathrm{~m}$ than $10 \sim 20 \mathrm{~m}$. Both graphs in the Figure 6 show relatively high pixel values at $10 \mathrm{~m} \sim 20 \mathrm{~m}$, but then show a nearly flat appearance. This suggests that there is an optimal altitude using drones for image acquisition. Above $100 \mathrm{~m}$, blurring was severe and edge detection was difficult, so image analysis was impossible.

\begin{tabular}{|c|c|c|c|c|c|c|c|c|}
\hline Altitude $(\mathrm{m})$ & GSD $(\mathrm{cm})$ & Point & RER & SNR & MTF(\%) & NIIRS & GRD(pixel) & GRD(cm) \\
\hline 10 & 0.195416 & 30 & 0.3398 & 110.745 & 6.7 & 11.9 & 2.3718 & 0.4635 \\
\hline 20 & 0.39083 & 30 & 0.5578 & 112.026 & 18.2 & 11.4 & 1.3710 & 0.5358 \\
\hline 30 & 0.586249 & 30 & 0.6264 & 119.133 & 22.1 & 11.0 & 1.3565 & 0.7953 \\
\hline 40 & 0.781665 & 30 & 0.6264 & 118.833 & 24.8 & 10.6 & 1.1198 & 0.8753 \\
\hline 50 & 0.977081 & 30 & 0.5865 & 116.929 & 18.4 & 10.2 & 1.3219 & 1.2916 \\
\hline 60 & 1.1725 & 30 & 0.5851 & 111.794 & 18.7 & 9.9 & 1.2825 & 1.5038 \\
\hline 70 & 1.36791 & 30 & 0.557 & 122.496 & 15.8 & 9.7 & 1.3696 & 1.8735 \\
\hline 80 & 1.56333 & 30 & 0.5619 & 121.013 & 19.3 & 9.5 & 1.3833 & 2.1626 \\
\hline 90 & 1.75875 & 30 & 0.5631 & 117.421 & 17.0 & 9.4 & 1.3606 & 2.3930 \\
\hline \multicolumn{2}{|c|}{ Average } & 30 & 0.5613 & 101.111 & 14.6 & 9.2 & 1.5187 & 2.9680 \\
\hline \multicolumn{2}{|r|}{} & 30 & 0.5566 & 115.1501 & 17.6 & 10.3 & 1.4456 & 1.4862 \\
\hline
\end{tabular}

Table 5. Image quality parameters in the Second drone shot images

\begin{tabular}{|c|c|c|c|c|c|c|c|c|}
\hline Altitude $(\mathrm{m})$ & GSD $(\mathrm{cm})$ & Point & RER & SNR & MTF(\%) & NIIRS & GRD(pixel) & GRD(cm) \\
\hline 10 & 0.195416 & 30 & 0.2219 & 86.28 & 5.02386 & 11.4 & 3.7182 & 0.7266 \\
\hline 20 & 0.39083 & 30 & 0.3473 & 82.79 & 6.00744 & 10.8 & 2.6291 & 1.0276 \\
\hline 30 & 0.586249 & 30 & 0.3413 & 94.10 & 5.78259 & 10.2 & 1.8620 & 1.0916 \\
\hline 40 & 0.781665 & 30 & 0.4559 & 105.30 & 7.14735 & 10.2 & 1.9643 & 1.5354 \\
\hline 50 & 0.977081 & 30 & 0.4745 & 111.4 & 8.31285 & 9.9 & 1.7937 & 1.7526 \\
\hline 60 & 1.1725 & 30 & 0.4849 & 89.96 & 8.96045 & 9.9 & 1.7917 & 2.1008 \\
\hline 70 & 1.36791 & 30 & 0.4947 & 116.77 & 7.57421 & 9.5 & 1.7980 & 2.4596 \\
\hline 80 & 1.56333 & 30 & 0.5261 & 101.53 & 11.5119 & 9.4 & 1.6127 & 2.5212 \\
\hline 90 & 1.75875 & 30 & 0.5113 & 161.58 & 9.29948 & 9.2 & 1.7679 & 3.1094 \\
\hline 100 & 1.95416 & 30 & 0.5249 & 100.14 & 10.6151 & 9.1 & 1.6141 & 3.1543 \\
\hline \multicolumn{2}{|r|}{ Average } & 29 & 0.4382 & 104.99 & 8.02352 & 9.9 & 2.0552 & 1.9479 \\
\hline
\end{tabular}

Table 6. Image quality parameters in the Third drone shot images

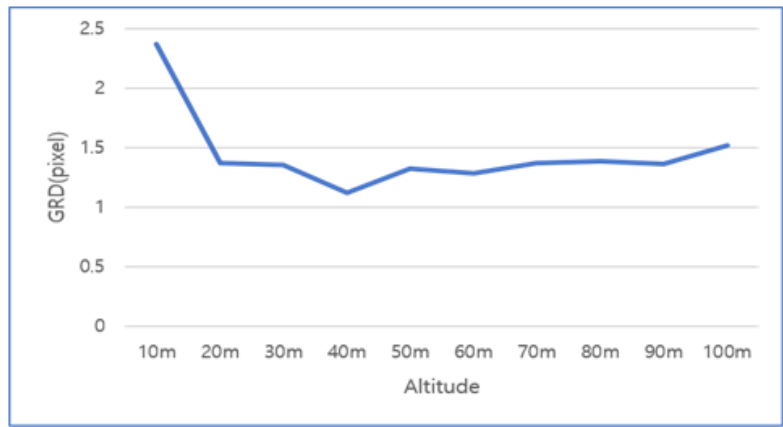

(a) GRD (pixel) by altitude in images of Second Drone shot

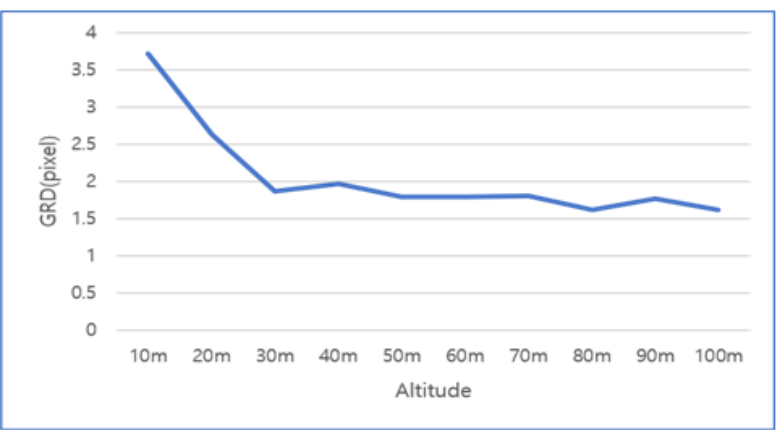

(b) GRD (pixel) by altitude in images of Second Drone shot

Figure7. GRD(pixel) in the Second and Third drone images 

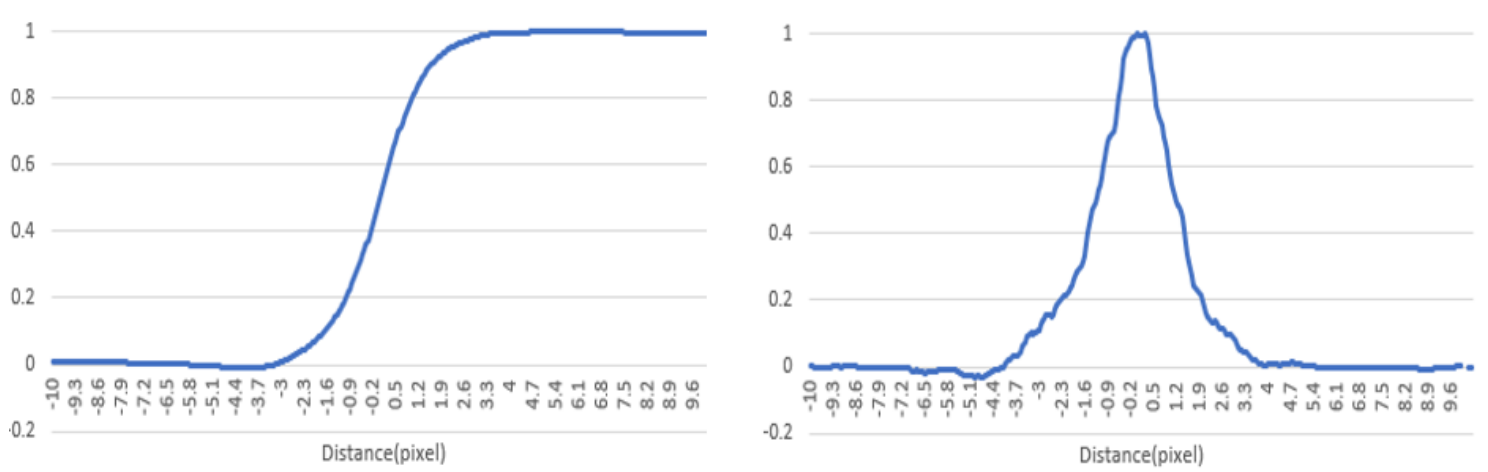

(a) Altitude 10m artificial target result
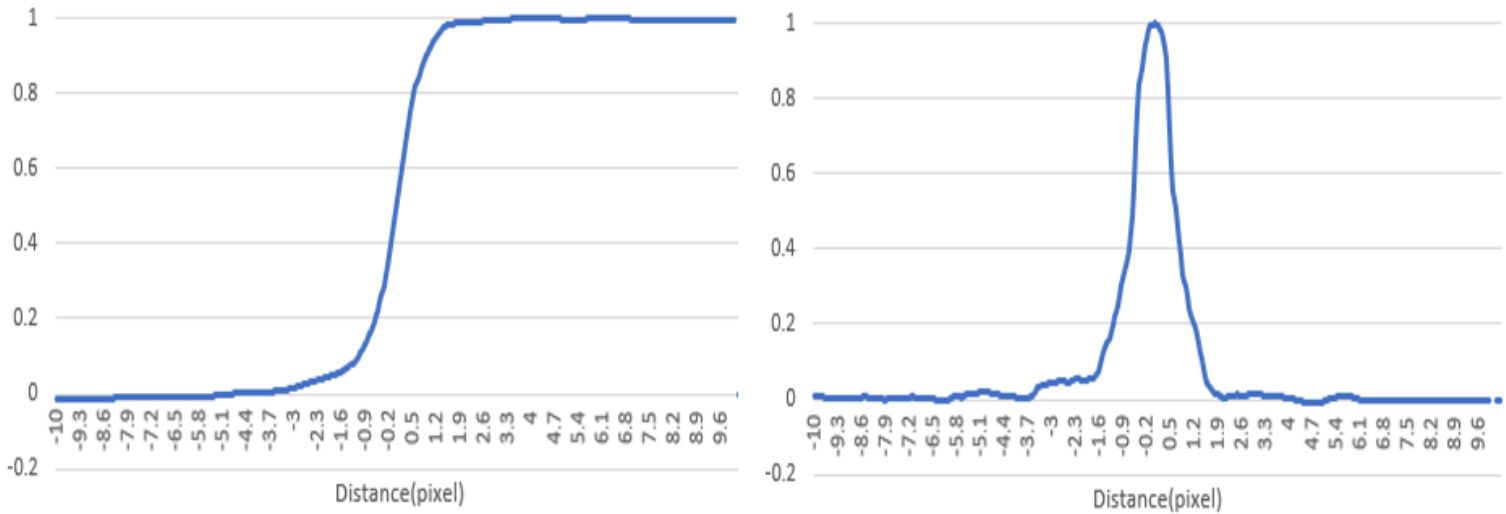

(b) Altitude $40 \mathrm{~m}$ artificial target result

Figure 8. Results of ESF and LSF calculation of Second drone shot images
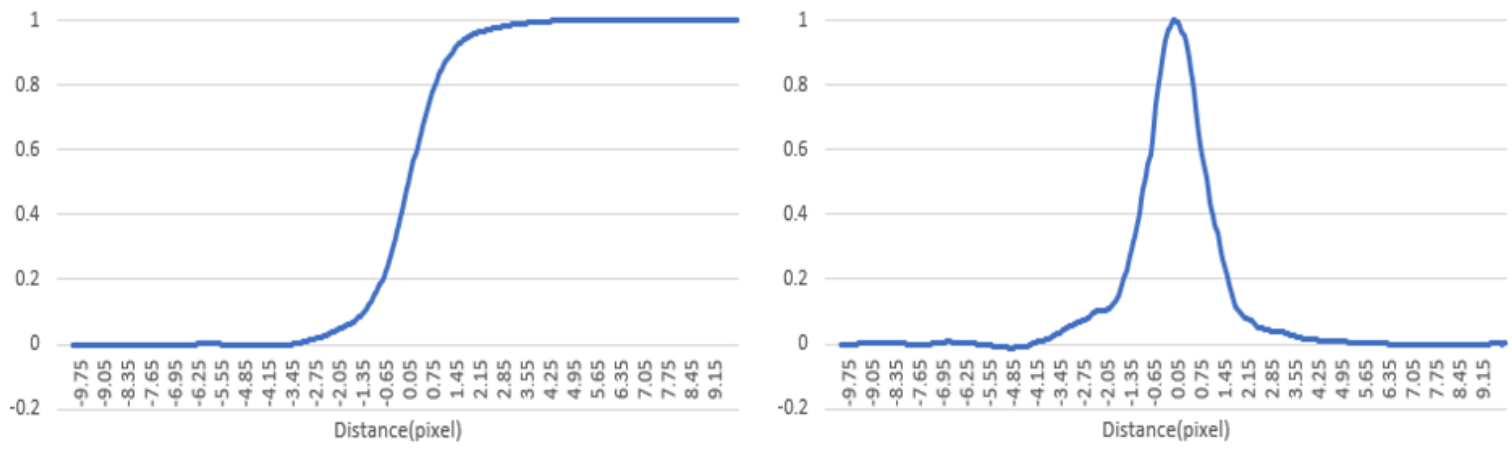

(a) Altitude $10 \mathrm{~m}$ artificial target result
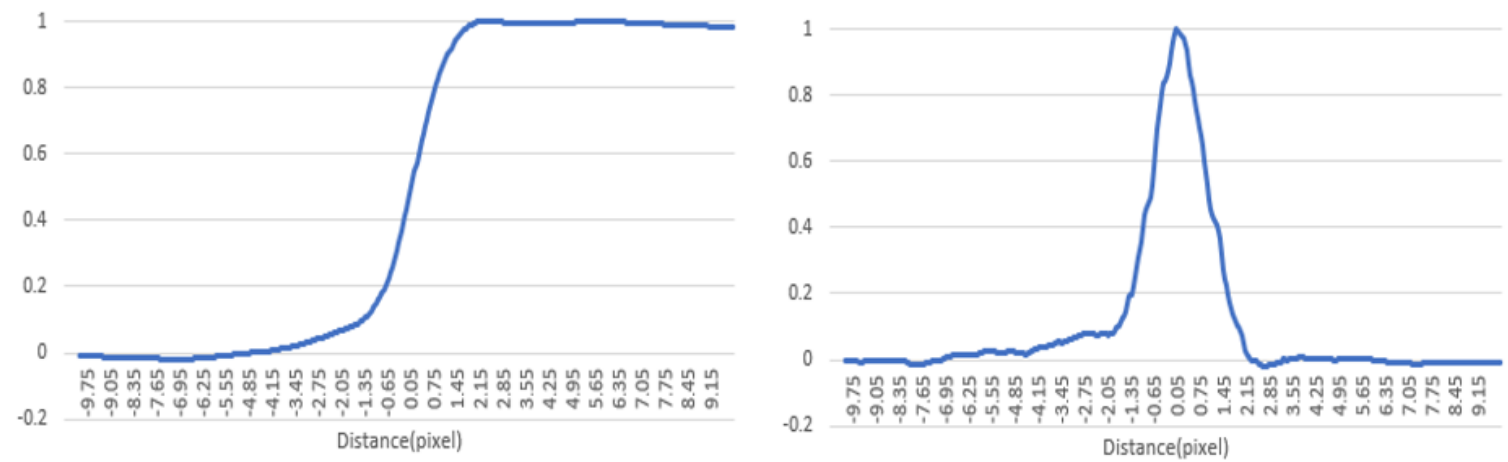

(b) Altitude $80 \mathrm{~m}$ artificial target result

Figure 9. Results of ESF and LSF calculation of Third drone shot images 


\section{CONCLUSION}

In this paper, the edge analysis method originally developed for satellite image was applied to UAV image to analyze image quality. To obtain reliable data, only artificial targets with clear brightness values were used. In the previous research, RER, SNR, MTF, NIIRS and GRD image quality parameters were calculated from artificial targets using self-developed software (Kim et al., 2010; Kim et al., 2011). Unlike satellite image cases image quality analysis using natural targets could not be attempted. Image quality analysis with artificial targets were affected greatly by many environmental factors.

Experiment images were acquired through a total of three times. The values of RER, SNR, MTF, and GRD were significantly different between images taken on the cloudy day and the sunny day. These values are believed to affect the weather. While the GSD and NIIRS values were depending on the altitude, GRD values in pixel were not. GRD, which represents image sharpness, showed a certain optimal range. The exact optimal range varied between images taken at different weather conditions. For the second image, which was experimented in the sunny weather, GRD was the best in the 30-80 m range rather than the low altitude range of $10-20 \mathrm{~m}$. The results of visual analysis were also similar. This implies that there is an altitude at which an optimum image can be obtained for each sensor and that edge analysis may be used to find such optimum range. The significance of this paper is that edge analysis can be applied to UAV image quality analysis. In the future, we plan to construct various shooting environments considering camera distortion and analyze image quality using various sensors.

\section{ACKNOWLEDGEMENTS}

This study was carried out with the support of "Cooperative Research Program for Agriculture Science \& Technology Development (PJ013500032018)" Rural Development Administration, Republic of Korea

\section{REFERENCES}

Campbell, J. B, 2002. INTRODUCTION TO REMOTE SENSING, Third Edition, Taylor \& Francis, London.

Leachtenauer J.C, MalilaW, IrvineJand ColburnL, 1997. "General Image-Quality Equation: GIQE", Applied Optics, Vol 36, Issue 32, pp. 8322 8328

Jae-In Kim, Jaehoon Jeong, and Taejung Kim, 2010, Development of GRD Measurement Method using Natural Target in Imagery, Korean Journal of Remote Sensing, Vol.26, No.5, pp.527 536

Jae-In Kim and Taejung Kim, 2011, Development of a Natural Target-based Edge Analysis Method for NIIRS Estimation, Korean Journal of Remote Sensing, Vol.27, No.5, pp.587 599 